\title{
Optical Identification and Monitoring of High Energy Gamma-Ray Sources
}

\author{
Steven D. Bloom* and Daniel A. Dale ${ }^{\Uparrow}$ \\ *Department of Physics and Astronomy, Hampden-Sydney College, Box 821, \\ Hampden-Sydney, VA, 23901 \\ ${ }^{I}$ Infrared Processing and Analysis Center, JPL/Caltech, Pasadena, CA, 91125
}

\begin{abstract}
We will be presenting updated results to our optical survey of the "error boxes" of EGRET unidentified sources at high Galactic latitude. It is our intention to search for potential blazars that may have been missed in the original identification process. We have first searched the error boxes of unidentified sources at $\mid \mathrm{bl}>20^{\circ}$ for flat spectrum radio sources using NASA Extragalactic Database (NED). For each such radio source found we conducted optical searches for counterparts using the Palomar 60-inch telescope. Many of the radio sources have plausible optical counterparts, and spectroscopy will be conducted at a later date to determine which sources are quasars or active galaxies (AGN's). Though it is plausible that several of these sources are extragalactic, no blazar-like activity has yet been observed.
\end{abstract}

\section{INTRODUCTION}

Though the EGRET instrument detected 271 sources, most of these sources remain unidentified with counterparts at another wavelength [7]. However, the distribution of sources on the sky does suggest at least two major components: Those near the Galactic plane, where $90 \%$ of the sources are unidentified, and those above the plane, where 50\% are unidentified [2]. Grenier [5] further suggests that those sources above the plane may have three subcomponents: extragalactic, Gould Belt, and Galactic halo. Though nearly all of the firmly identified extragalactic sources are blazars, there have been recent studies suggesting that some sources may be related to galaxy clusters [3]. The task of investigating all of these sources individually seems daunting. Therefore, several investigators have gone the route of examining several individual sources in depth ([6], [11], [13]). The advantage of this technique is that such searches are much more likely to be fruitful. This has particularly been true for finding new blazar-like objects at high Galactic latitude. We continue along these lines by searching within the error contours of high Galactic latitude EGRET unidentified sources for new blazars. We will then ascertain whether any such objects found are likely to be the source of the gamma rays.

CP587, GAMMA 2001: Gamma-Ray Astrophysics 2001, edited by S. Ritz et al. (C) 2001 American Institute of Physics 0-7354-0027-X/01/\$18.00 


\section{SOURCE SELECTION}

Using the NASA Extragalactic Database (NED), we have searched for flat spectrum radio sources (spectral index, $-0.5<\alpha<0.5$ ) within the $95 \%$ error contours of the EGRET unidentified sources with $|\mathrm{b}|>20^{\circ}$. Aside from a handful of pulsars, blazars with flat radio spectra are the only objects that have been detected by EGRET as a class. We have chosen this range of latitudes so that there will be minimal overlap with Galactic objects. We further restricted ourselves to the ranges of right ascension and declination that would be visible from Mount Palomar during the nights in November 1999. These radio data referenced in NED were usually from the Parkes or Green Bank surveys, which had positional accuracies of about 1-3 arc minutes (depending on observing frequency and brightness of the source), which is far too coarse for optical searches. We have therefore additionally searched for these radio sources in the NRAO-VLA Sky Survey (NVSS) in order to obtain positions accurate to approximately 1" [4]. In addition to the flat spectrum radio sources, we have compiled a secondary observing list consisting of radio sources of unknown spectral index, and/or X-ray and optical sources with accurate positions.

An extensive study of the identification of radio sources with EGRET sources was conducted by Mattox et al. [10]. Essentially they conclude that it is difficult to positively identify a gamma-ray source with any radio source with flux density less than about $500 \mathrm{mJy}$ at $5 \mathrm{GHz}$ mostly because the sky density of such sources is so high, and thus the probability for chance correlations is correspondingly high. They assume that the radio spectra would be near their peak fluxes at these frequencies, and thus $5 \mathrm{GHz}$ flux densities would be representative of the synchrotron output of the source. This assumption was made out of necessity, since there are no complete high frequency surveys with sensitivity less than one Jansky. However, other investigators have shown that several sources might be fairly dim at these low frequencies, and have a brighter (and flatter) spectrum extending to at least $200 \mathrm{GHz}$ (eg., [1]). Thus, some $\operatorname{dim} 5 \mathrm{GHz}$ radio sources, which by the method of [10] would not be considered to be counterparts to EGRET sources, may indeed be counterparts to gamma-ray blazars. We intend to make up for this possible deficiency by searching for the optical counterparts to sources that may be fairly $\operatorname{dim}(<100 \mathrm{mJy})$ at $5 \mathrm{GHz}$. If a counterpart is found we will search for blazar-like activity, and go on to investigate the optical and radio spectra of these objects.

\section{OBSERVATIONS}

We have conducted the search for optical counterparts using the 60 -inch telescope at Mount Palomar with a Tek $2048 \times 2048$ CCD. The pixel size is 0.37 arc seconds, however we have $2 \times 2$ binned the pixels to a size of 0.74 arc seconds. Since the seeing for those nights (and most at Palomar) was worse than 1 arc second, no crucial information on source structure is lost. In addition, we would expect most of our sources to be point-like. We have used the Johnson B, V and Kron R filters for determining magnitudes in those bandpasses. The procedure was to first observe a candidate source in $\mathrm{R}$ for 10 minutes (and then adjust to a shorter or longer exposure depending on results). If no obvious counterpart was seen after 20 minutes, we did not 
go to the other filters. If a potential source was seen, we observed in $\mathrm{V}$ and $\mathrm{B}$ as well. In addition to the program sources, we have observed Landolt [8] standard stars throughout the evening to determine the photometric scale and atmospheric absorption in the usual manner (more details on procedure and reductions will appear in a forthcoming journal article by the authors). Galactic extinction corrections have been applied using the values from NED. We have repeated our observations on a second night to test for variability. We have used the Guide Star Catalogue to determine the astrometric plate solutions for our CCD fields. Typical rms uncertainties in our plate solutions were less than that of the individual stellar positions from the catalogue (about 0.3 arc seconds).

\section{RESULTS}

We have observed 10 radio sources (within four different EGRET error boxes) and found optical counterparts for seven, breaking into magnitude in the following manner. Of the seven sources seen visually, five are in the range of $V=16-19$, one is in the range of $\mathrm{V}=19-21$ and one is dimmer than 21 (near the magnitude limit). A summary of the results for the visually detected sources is in Table 1. A more complete summary with multicolor photometry will be presented in a forthcoming paper. Column (1) gives the name of the EGRET source, Column (2) gives the name of the radio source that is a candidate counterpart, Column (3) gives the V magnitude attained by us as described in the previous section, Column (4) give the radio flux density at $5 \mathrm{GHz}$ (in milliJanskys) as found in NED and Column (5) gives the spectral index between 1.4 and $5 \mathrm{GHz}$ and is provided by NED if the information is known. We note that no source has shown significant variability across the two nights. Of particular interest is that two candidate counterparts (B0214+108 and NVSS J032850+21282) are in the catalogue of ROSAT/Green Bank ("RGB") sources [9]. Punsly [12] suggested that "RGB" sources would be likely gamma-ray blazars and that the counterparts are BL Lac objects with their spectral energy distributions (SED) peaked at intermediate to high frequencies (that is, optical to X-ray). These particular objects in our study, however, do not have well determined SED's. The brighter objects have been slated for spectroscopic and high frequency radio observations within the next several months (to be conducted by other groups, in collaboration with us in this study).

TABLE 1. Possible Counterparts to Gamma-Ray Sources

\begin{tabular}{|l|l|l|l|l|}
\hline EGRET Source & Radio Source & V & Radio & S.I. \\
\hline (1) & $\mathbf{( 2 )}$ & $\mathbf{( 3 )}$ & $\mathbf{( 4 )}$ & $\mathbf{( 5 )}$ \\
\hline 3EG J0038-0949 & NVSS J003906-094247 & 21.5 & 214 & -0.1 \\
\hline 3EG J0215+1123 & NVSS J021527+112318 & 17.9 & 56 & -0.8 \\
\hline 3EG J0215+1123 & B0214+108 & 16.3 & 440 & -0.8 \\
\hline 3EG J0245+1758 & NVSS J024437+172221 & 19.8 & 33 & -0.1 \\
\hline 3EG J0245+1758 & NVSS J024640+180144 & 18.1 & 53 & $\ldots$ \\
\hline 3EG J0245+1758 & NVSS J024611+182330 & 18.8 & 214 & 0.1 \\
\hline 3EG J0329+2149 & NVSS J032850+212825 & 18.1 & 57 & $\ldots$ \\
\hline
\end{tabular}




\section{CONCLUSIONS}

After examining the error boxes of four EGRET sources for coincident radio/optical objects, we have found four plausible counterparts to the gamma-ray sources. A summary of the results is shown in Table 1. For 3EG J0038-0949 there is only one plausible counterpart. For 3EG J0215+1123, we consider B0214+108 to be the most plausible counterpart, though even this source has difficulties. The extended radio source, 4C +10.06 is ambiguously identified with this catalogued QSO. The two nearest compact NVSS radios source are over 40 arc seconds from the optical object. Search results from NED indicate that there are many galaxies in this field, at least one of which has a red shift similar to the QSO. For 3EG J0245+1758, the second NVSS source listed in the table is the more likely counterpart (brighter, flat spectrum). This source is also a known QSO. At first, it may not seem that the suggested counterpart for 3EG J0329+2149 is plausible, due to its relatively low radio flux density; however, we note that this source is a coincident optical, IR, radio, and X-ray source. The compact core is known to have a flat spectrum [9]. We emphasize that we do not yet consider any of these suggestions to be firm identifications. We suggest that optical monitoring of these objects continue, and that spectroscopy conducted for any of the objects for which the optical counterpart has not yet been classified spectrally. The Hampden-Sydney College 16 inch telescope could be used to monitor the brightest 5 or so of these sources when they next become visible in northern Autumn 2001.

\section{ACKNOWLEDGMENTS}

This work has made use of the NASA Extragalactic Database and the Astrophysical Data System web abstract server.

\section{REFERENCES}

1. Bloom, S. D. et al., Ap. J., 488, L23 (1997)

2. Caraveo, P., these proceedings (2001)

3. Colafrancesco, S., these proceedings (2001)

4. Condon, J. J. et al., A. J., 115, 1693 (1998)

5. Grenier, I., these proceedings (2001)

6. Halpern, J. et al., Ap. J, 551, 1016 (2001)

7. Hartman, R. C. et al., Ap. J. Suppl. 123, 79 (1999)

8. Landolt, A. U., A. J., 104, 340 (1992)

9. Laurent-Muelheisen, S. A. et al., A\&A Suppl., 122, 235 (1997)

10. Mattox, J. R. et al., Ap. J., 481, 95 (1997)

11. Mukherjee, R. et al., Ap J, 542, 740 (2000)

12. Punsly, B., Ap. J., 516, 141(1999)

13. Wallace, P. M., these proceedings (2001) 